\title{
Left ventricular rotational abnormalities in adult patients with corrected tetralogy of Fallot following different surgical procedures (Results from the CSONGRAD Registry and MAGYAR-Path Study)
}

\author{
Attila Nemes, Gergely Rácz, Árpád Kormányos, Péter Domsik, Anita Kalapos, Nándor Gyenes, \\ Nóra Ambrus, István Hartyánszky, Gábor Bogáts, Kálmán Havasi \\ 2nd Department of Medicine and Cardiology Centre, Medical Faculty, Albert Szent-Györgyi Clinical Center, University of Szeged, Szeged, Hungary \\ Contributions: (I) Conception and design: A Nemes; (II) Administrative support: N Ambrus; (III) Provision of study materials or patients: A Nemes, \\ K Havasi; (IV) Collection and assembly of data: G Rácz, Á Kormányos, P Domsik, A Kalapos; (V) Data analysis and interpretation: G Rácz, Á \\ Kormányos, A Nemes; (VI) Manuscript writing: All authors; (VII) Final approval of manuscript: All authors. \\ Correspondence to: Attila Nemes, MD, PhD, DSc, FESC. 2nd Department of Medicine and Cardiology Center, Medical Faculty, Albert Szent-Györgyi \\ Clinical Center, University of Szeged, H-6725 Szeged, Semmelweis Street 8, P.O. Box 427, Hungary. Email: nemes.attila@med.u-szeged.hu.
}

Background: Tetralogy of Fallot (TOF) is the most common cyanotic congenital heart disease (CHD). Abnormal aortic dimensions and elasticity parameters have been long described for corrected TOF (cTOF) together with left ventricular (LV) rotational abnormalities, but results are conflicting. The present study focuses on investigating $\mathrm{LV}$ rotational mechanics in cTOF, and possible correlation of these parameters with aortic elasticity. It was also aimed to be examined whether different surgical strategies have any effect on these results.

Methods: The study involved 26 adult cTOF patients, from which 14 had palliative surgery first and a late total correction (pcTOF), while early total correction was the treatment of choice in 12 patients (etrTOF). Their results were compared to those of 37 age- and gender-matched healthy adults. Routine transthoracic two-dimensional Doppler echocardiography extended with assessment of aortic elastic properties and threedimensional speckle-tracking echocardiography (3DSTE) was performed in all cTOF patients and controls.

Results: Sixteen out of 26 cTOF patients showed normally directed LV rotational mechanics, while apical or basal LV rotations were in the same clockwise or counterclockwise directions in 7 and 3 cTOF cases, respectively (LV 'rigid body rotation', RBR). Significantly reduced LV apical rotation and twist could be demonstrated in all cTOF patients with preserved LV basal rotation regardless of previous procedure. pcTOF patients showed significantly reduced LV apical rotation as compared to that of etrTOF cases. Significant correlations could be demonstrated between $L V$ apical rotation and aortic stiffness index $(r=-0.55$, $\mathrm{P}=0.03)$ and aortic distensibility $(\mathrm{r}=0.52, \mathrm{P}=0.04)$.

Conclusions: Significant LV rotational abnormalities could be demonstrated in cTOF with the high prevalence of LV-RBR. pcTOF patients showed significantly reduced LV apical rotation as compared to that of etrTOF cases. Unexpected abnormal physiologic response of $\mathrm{LV}$ rotational mechanics to increased aortic stiffness can be detected in cTOF patients without LV-RBR.

Keywords: Fallot; aortic stiffness; three-dimensional; echocardiography; rotation

Submitted Mar 17, 2020. Accepted for publication May 28, 2020.

doi: $10.21037 / \mathrm{cdt}-20-365$

View this article at: http://dx.doi.org/10.21037/cdt-20-365 


\section{Introduction}

Tetralogy of Fallot (TOF) is the most common (3-5\%) cyanotic congenital heart disease (CHD), corresponding to one in 3,600 live births (1). The survival for untreated TOF was quite poor, but now it has a good prognosis due to timely follow-ups of residua, complications and justin-time tailored interventions (1). Due to advances in the surgical treatment of TOF in the last decades, almost all patients with correction (cTOF) can now expect to survive to adulthood (1). Dilated aortic dimensions and impaired elasticity have been long-described for cTOF $(2,3)$. Moreover, left ventricular (LV) rotational abnormalities are known features in cTOF, but the results are conflicting $(4,5)$. Although theoretically several factors can affect aortic stiffness and LV rotational mechanics in cTOF at the same time, their relationship has never been assessed. Therefore, the present study focuses on investigating LV rotational abnormalities in cTOF, and the possible correlation between these parameters and increased aortic stiffness. It was also aimed to be examined whether different surgical strategies have any effect on these results. We present the following article in accordance with the STROBE reporting checklist (available at http://dx.doi.org/10.21037/cdt-20-365).

\section{Methods}

\section{Patient population}

The study prospectively involved 26 adult cTOF patients without pacemaker, who were willing to participate in this study. All data of these patients originate from the Registry of C(S)ONGenital caRdiAc Disease patients at the University of Szeged (CSONGRAD Registry), which manages data of more than 3,000 CHD patients since 1961, who were treated and/or operated on at the Department of Pediatrics, Department of Heart Surgery, and 2nd Department of Medicine and Cardiology Center at the University of Szeged, Hungary (6). From this cohort, 247 had diagnosis of TOF (died 57) and 50 are under regular follow-up. Their results were compared to those of 37 age- and gendermatched healthy adults (Table 1). Routine transthoracic two-dimensional Doppler echocardiography extended with assessment of aortic elastic properties and three-dimensional speckle-tracking echocardiography (3DSTE) was performed in all cTOF patients and controls. This study is a part of the Motion Analysis of the heart and Great vessels bY three-dimensionAl speckle-tRacking echocardiography in Pathological cases Study (MAGYAR-Path Study) organized at the Cardiology Center of the University of Szeged, which aimed to assess diagnostic and prognostic values of 3DSTE-derived variables in different CHDs among others (7). Informed consent was given by both cTOF patients and control subjects. The institutional human research committee approved the study, which complied with the 1975 Declaration of Helsinki (project number: 71/2011).

\section{Transthoracic echocardiography}

Echocardiographic examinations were performed with a Toshiba Artida ${ }^{\mathrm{TM}}$ cardiac ultrasound system (Toshiba Medical Systems, Tokyo, Japan) with a PST-30SBP 1-5 MHz phased-array transducer. The experts performing analysis were blinded to the clinical data. From parasternal long axis-view using M-mode echocardiography LV diameters, volumes and ejection fraction, left atrial and aortic dimensions were assessed. Right ventricular (RV) function was characterized by tricuspid annular plane systolic excursion (TAPSE) and RV fractional area change (RV-FAC). The measurements were averaged from 3 separate heart cycles (8). Valvular regurgitations were visually scored, valvular stenosis was excluded by Doppler echocardiography.

\section{Measurement of blood pressure values}

In supine position, and with the cuff applied to the right arm after 15 minutes resting systolic and diastolic blood pressures (SBP and DBP, respectively) were measured. For SBP and DBP the first and fifth Korotkoff sounds were considered. Caffeine or nicotine substances were not consumed prior to the measurements (9).

\section{Evaluation of aortic stiffness parameters}

In parasternal long-axis view, $3 \mathrm{~cm}$ above the level of the aortic valve, $M$-mode images were recorded, and systolic and diastolic ascending aortic diameters were measured (SD and DD) at the time of maximum aortic anterior motion and at the peak of the QRS complex, respectively (9). For better accuracy, 3 measurements were repeated. The aortic elastic properties were calculated as follows:

$$
\begin{aligned}
& \text { Aortic strain }(\mathrm{AS})=(\mathrm{SD}-\mathrm{DD}) / \mathrm{DD} ; \\
& \text { Aortic distensibility }(\mathrm{AD})=2 \times(\mathrm{SD}-\mathrm{DD}) /[(\mathrm{SBP}- \\
& \mathrm{DBP}) \times \mathrm{DD}] ; \\
& \text { Aortic stiffness index }(\mathrm{ASI})=\ln (\mathrm{SBP} / \mathrm{DBP}) /[(\mathrm{SD}- \\
& \mathrm{DD}) / \mathrm{DD}], \text { ('ln' being the natural logarithm). }
\end{aligned}
$$


Table 1 Comparison of demographic, two-dimensional echocardiographic data and aortic elastic properties in patients with tetralogy of Fallot and in controls

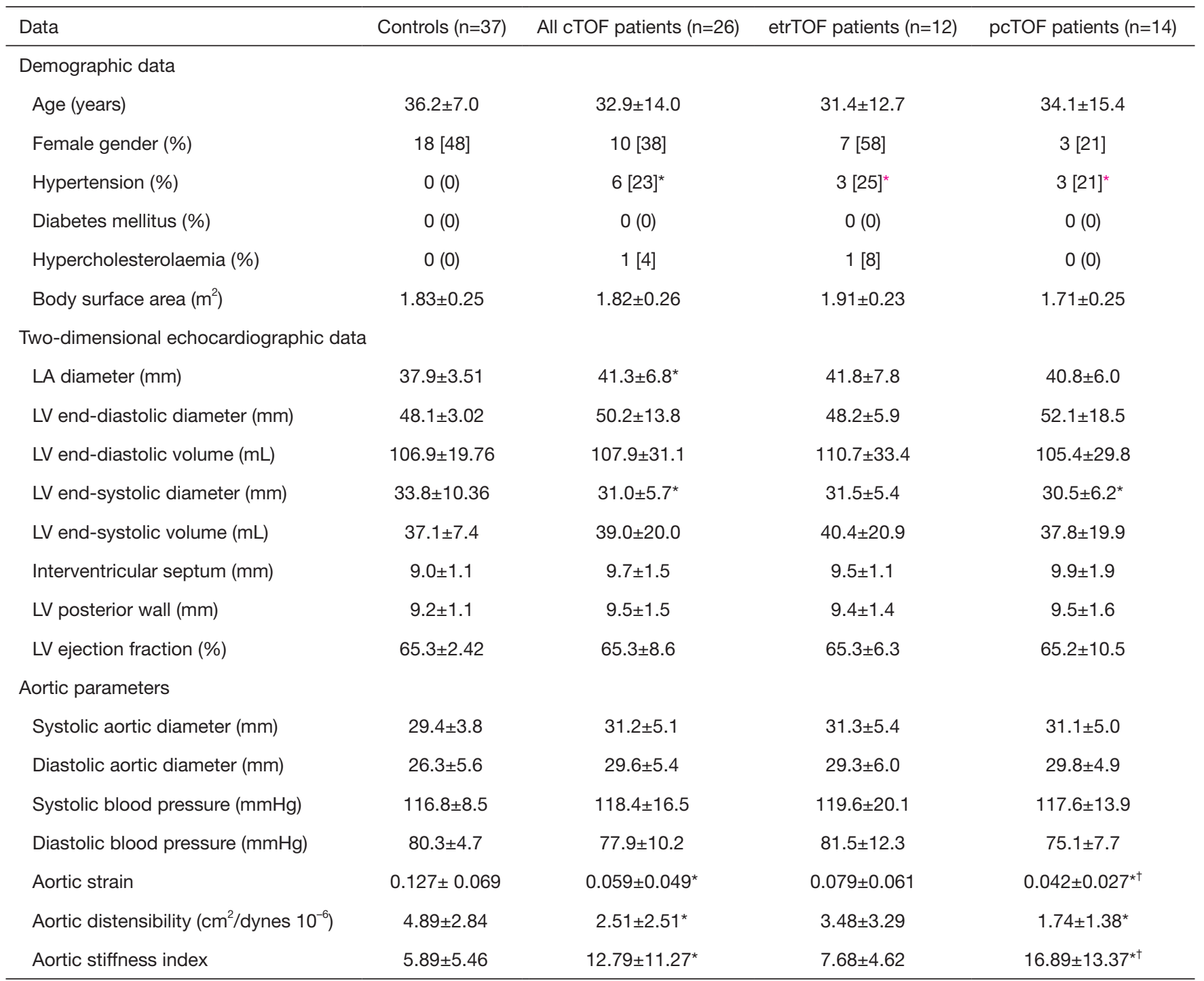

*, $\mathrm{P}<0.05$ vs. controls; ${ }^{\dagger}, \mathrm{P}<0.05$ vs. etrTOF. cTOF, corrected tetralogy of Fallot; etrTOF, early total reconstruction for patients with TOF; pcTOF, early palliation, late correction for patients with TOF; LA, left atrial; LV, left ventricular.

\section{Three-dimensional speckle-tracking echocardiography}

Toshiba Artida ${ }^{\mathrm{TM}}$ echocardiographic tool (Toshiba Medical Systems, Tokyo, Japan) using a 1-4 MHz matrix phasedarray PST-25SX was used to perform the 3DSTE studies with the transducer placed in apical window (7). From the acquired 6 wedge-shaped subvolumes, a full pyramid-shaped dataset was generated, with constant RR interval monitored by ECG gating. Using 3D Wall Motion Tracking software version 2.7 (Toshiba Medical Systems) quantifications were performed. From the 3D echocardiographic 'cloud' apical two- $(\mathrm{AP} 2 \mathrm{CH})$ and four-chamber $(\mathrm{AP} 4 \mathrm{CH})$ views and 3 short-axis views at different $\mathrm{LV}$ levels were automatically reconstructed (Figure 1). On the $\mathrm{AP} 2 \mathrm{CH}$ and $\mathrm{AP} 4 \mathrm{CH}$ views the region of interest was then manually marked, with points at the bases of the $\mathrm{LV}$ at the mitral annular edges, and at the LV apex. The reconstructed endocardial surface was then tracked during the cardiac cycle creating a virtual $3 \mathrm{D}$ cast of the $\mathrm{LV}$. Using this $3 \mathrm{D} \mathrm{LV}$ model, $\mathrm{LV}$ rotational parameters (apical and basal LV rotation and their absolute 

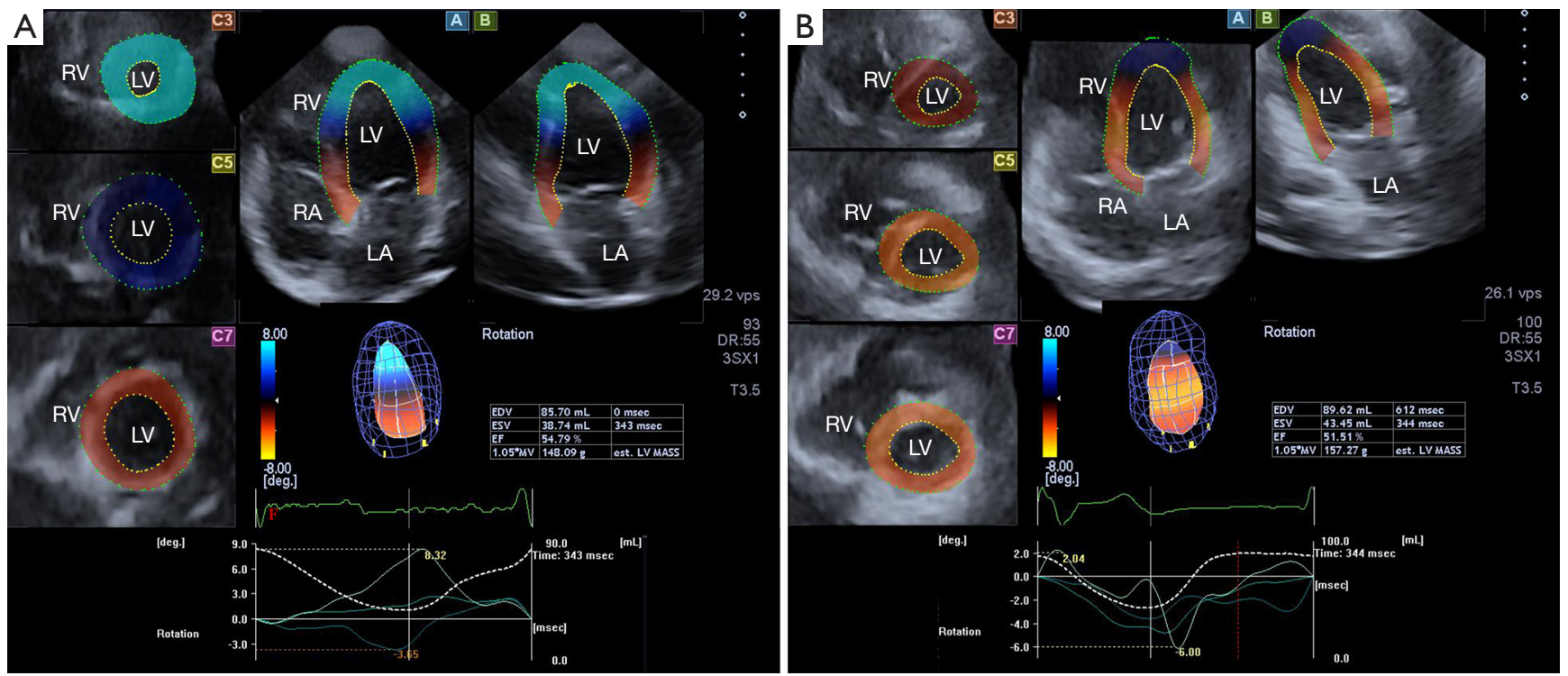

Figure 1 Three-dimensional (3D) speckle tracking examination of left ventricular (LV) rotational mechanics is presented in a patient with normally directed rotational mechanics (Panel A) and in a subject with apical and basal LV rotations in the same counterclockwise direction (LV 'rigid body rotation') (Panel B). Apical four-chamber (A) and two-chamber (B) LV views, cross-sectional views at different levels of the LV (C3, C5, C7), virtual 3D model of the LV and LV volumetric data are shown in both (Panel A) and (Panel B). Actual LV apical (white line), midventricular (light blue line) and basal (dark blue line) LV rotational curves and LV volume changes during a cardiac cycle (dashed line) are also demonstrated.

sum called LV twist) were calculated respecting the cardiac cycle. If apical and basal LV rotations were in the same direction, LV apico-basal gradient was measured.

\section{Statistical analysis}

The authors reported continuous variables as mean values \pm standard deviation. Categorical data are presented as frequencies and percentages. To compare subgroups, Student $t$ test, $\chi^{2}$ test, and Fisher's exact test, when appropriate, were performed. The results were considered statistically significant when the $\mathrm{p}$ value was less than 0.05. To determine whether numerical correlations are present, Spearman correlation coefficient was calculated. For statistical analysis, Medcalc software version 13.1.2.0 (MedCalc, Mariakerke, Belgium) was used.

\section{Results}

\section{Clinical, two-dimensional echocardiographic and aortic stiffness data}

From the 26 adult cTOF patients 14 had palliative surgery first [modified Blalock-Taussig ( $\mathrm{n}=10)$, Waterston-Cooley $(n=2)$ shunts, or Brock procedure $(n=2)$ ] (mean age at the palliation: $2.3 \pm 2.3$ years, median age: 1.5 years, interquartile range: 3.5 years) and a late total correction (mean age at the total correction: $11.6 \pm 13.1$ years, median age: 7.0 years, interquartile range: 6.0 years) (pcTOF), while the early total correction was the treatment of choice in 12 patients (mean age at the repair: $4.2 \pm 3.1$ years, median age: 5.0 years, interquartile range: 5.3 years) (etrTOF). Data are presented in Table 1. One quarter of cTOF patients showed hypertension regardless of previous procedure. Dilated left atrium and smaller LV end-systolic diameter could be demonstrated in cTOF patients. The TAPSE and RV-FAC values of cTOF patients were $17.9 \pm 4.7 \mathrm{~mm}$ and $35.1 \% \pm 3.3 \%$, respectively. Significant ( $>$ grade 2 ) tricuspid regurgitations (TR) could be detected in $4(15.4 \%)$ cTOF patients. None of the cTOF patients showed significant mitral and pulmonary $(\mathrm{PR})$ regurgitation. Mean RV pressure was $14.8 \pm 2.3 \mathrm{mmHg}$ without residual pulmonary stenosis or branch pulmonary artery narrowing. Most of our patients had normal aortic dimensions for adults with cTOF, and only 3 had larger than $35 \mathrm{~mm}$, but less than $40 \mathrm{~mm}$ systolic aortic diameter. Reduced AS and $\mathrm{AD}$ and increased ASI could be detected in cTOF patients as compared to controls. When etrTOF and pcTOF patients 
Table 2 Comparison of three-dimensional speckle-tracking echocardiography-derived left ventricular rotational mechanics in patients with tetralogy of Fallot and in controls

\begin{tabular}{|c|c|c|c|c|}
\hline Data & Controls $(n=37)$ & All cTOF patients $(n=26)$ & etrTOF patients $(n=12)$ & pcTOF patients $(n=14)$ \\
\hline Clockwise LV-RBR (\%) & $0(0)$ & $7[27]^{*}$ & $2[16]$ & $5[35]^{*}$ \\
\hline Counterclockwise LV-RBR (\%) & $0(0)$ & $3[11]$ & $2[16]$ & $1[7]$ \\
\hline \multicolumn{5}{|l|}{ Normally directed LV rotational mechanics $(n=16)$} \\
\hline LV apex rotation (degree) & $10.2 \pm 4.5$ & $5.2 \pm 3.7^{*}$ & $7.1 \pm 2.5^{\star}$ & $3.2 \pm 3.7^{\star} \dagger$ \\
\hline LV twist (degree) & $14.6 \pm 4.9$ & $9.0 \pm 3.3^{*}$ & $10.5 \pm 2.8^{*}$ & $7.6 \pm 3.2^{*}$ \\
\hline LV twist time (ms) & $337 \pm 94$ & $361 \pm 108$ & $321 \pm 69.5$ & $402 \pm 128$ \\
\hline
\end{tabular}

*, $\mathrm{P}<0.05$ vs. controls; ${ }^{\dagger}, \mathrm{P}<0.05$ vs. etrTOF. cTOF, corrected tetralogy of Fallot; etrTOF, early total reconstruction for patients with TOF; pcTOF, early palliation, late correction for patients with TOF; LV, left ventricular; 3DSTE, three-dimensional speckle-tracking echocardiography; RBR, rigid body rotation.

were compared, etrTOF patients showed more beneficial aortic stiffness parameters than pcTOF patients.

\section{DSTE-derived LV rotational mechanics}

Sixteen out of 26 cTOF patients showed normally directed $\mathrm{LV}$ rotational mechanics, while apical and basal LV rotations were in the same clockwise or counterclockwise directions in 7 and 3 cTOF cases, respectively (10 out of 26 , $38 \%$ ) (Table 2). This sort of $\mathrm{LV}$ movement is called as $\mathrm{LV}$ rigid body rotation (RBR). The ratio of $L V-R B R$ between etrTOF and pcTOF patients did not differ significantly ( $33 \%$ vs. $43 \%, \mathrm{P}=0.70$ ). cTOF patients with $\mathrm{LV}-\mathrm{RBR}$ were managed separately during assessments.

\section{Normally directed $L V$ rotational mechanics}

Significantly reduced LV apical rotation and twist could be demonstrated in all cTOF patients with preserved LV basal rotation regardless of previous procedure (Table 2). pcTOF patients showed significantly reduced $\mathrm{LV}$ apical rotation as compared to that of etrTOF cases.

\section{$L V$ rigid body rotation}

From the 7 cTOF patients with clockwise LV-RBR, apical and basal $L V$ rotation proved to be $-6.8 \pm 5.0$ and $-4.8 \pm 2.3$ degrees, respectively with $L V$ apico-basal gradient of $4.3 \pm 3.3$ degrees. Apical and basal LV rotations of particular etrTOF patients were -12.3 and -8.5 degrees, -5.1 and -2.0 degrees, respectively, while the same values of pcTOF patients proved to be -8.5 and -5.5 degrees, -1.6 and -6.9 degrees, -3.9 and -5.4 degrees, -0.9 and -2.2 degrees, -15.2 and -3.4 degrees, retrospectively. The mean $\mathrm{LV}$ apical and basal $\mathrm{LV}$ rotation of 3 cTOF patients with counterclockwise LV-RBR proved to be $2.6 \pm 2.3$ and $0.7 \pm 0.3$ degrees, respectively with apico-basal gradient of $1.9 \pm 2.4$ degrees. Apical and basal LV rotations of etrTOF patients were 5.9 and 0.7 degrees, 1.2 and 1.1 degrees, respectively, while the same values of the pcTOF patients proved to be 0.7 and 0.4 degrees. In this cohort of patients with LV-RBR, LV apico-basal gradient had no relationship with QRS duration, LA size, LV diastolic parameters, hypertension, arrhythmia, exercise capacity, TR and PR.

\section{Correlations}

Significant correlations could be demonstrated between LV apical rotation and aortic stiffness index $(\mathrm{r}=-0.55, \mathrm{P}=0.03)$ and aortic distensibility $(\mathrm{r}=0.52, \mathrm{P}=0.04)$ in all cTOF patients with normally directed $\mathrm{LV}$ rotational mechanics. No further correlations could be demonstrated between any other aortic elastic properties and LV rotational parameters in all cTOF groups and during subgroup analyses. No correlations were seen according to right bundle branch block or QRS-duration in ECG.

\section{Discussion}

In spite of rapid development in cardiovascular imaging, 
echocardiography is still the basic imaging modality due to its non-invasiveness, relatively good image quality, reproducibility and short learning-curve $(7,8)$. Moreover, it allows simultaneous assessment of cardiovascular abnormalities like aortic elastic properties by M-mode echocardiography and LV rotational mechanics by 3DSTE $(7,9)$. Aortic stiffness describes elasticity of the aorta which sets against its distension (9). In normal situations, basal and apical LV regions rotate in opposite directions: while $\mathrm{LV}$ apex rotates in counterclockwise direction, $\mathrm{LV}$ base rotates in clockwise direction resulting in a 'towel-wringing motion'-like movement called LV twist (10). It is known that aortic stiffness correlates with $\mathrm{LV}$ rotational parameters in healthy subjects, larger aortic stiffness is associated with increased LV apical rotation and twist suggesting an early compensating mechanism (11). Moreover, both increased aortic stiffness (3) and $L V$ rotational abnormalities $(4,5)$ could be detected in cTOF. However, their relationship and the effect of different treatment strategies on these parameters have never been investigated.

According to the present findings, it could be stated that high ratio of cTOF patients showed LV-RBR (38\%). In a recent study by Menting et al., 11 and 2 out of 82 (13\% and $2 \%$, respectively) cTOF patients had reversed or absent $\mathrm{LV}$ apical rotation (clockwise LV-RBR), while 9 and 6 out of 82 (11\% and 7\%, respectively) showed reversed or absent $L V$ basal rotation (counterclockwise LV-RBR) (4). The same parameters proved to be $27 \%$ and $11 \%$ in the present study with a smaller population. In the study of Dragulescu et al., 19 of 50 young cTOF patients had reversed (counterclockwise) LV basal rotation (5). According to the literature, LV-RBR was found to be a frequent phenomenon in several disorders with the largest ratio in non-compaction (50-100\%) (12), dilated (50\%) (13) and hypertensive cardiomyopathies (32\%) (14), cardiac amyloidosis (60\%) (15) and acromegaly (20\%) (16). Some case reports showed LV-RBR in some CHDs as well including Ebstein's anomaly (17), univentricular heart (18) and hypoplastic right-heart syndrome (19).

Eight out of $82(10 \%)$ cTOF patients had reduced LV apical rotation in the study by Menting et al. (4). In our study, the majority of cTOF patients had no LV-RBR (62\%). Although direction of $\mathrm{LV}$ rotational mechanics proved to be normal in these patients, $L V$ apical rotation was significantly reduced as compared to that of controls. Moreover, the impairment proved to be more pronounced in pcTOF patients. $L V$ apical rotation was reduced, but not reversed in the study of Dragulescu et al. (5). In our study, LV basal rotation proved to be preserved. According to the results of van Grootel et al., reduced $L V$ apical rotation is associated with worse outcomes in cTOF patients (20).

In the present study, significantly impaired aortic elastic properties could be demonstrated in cTOF patients in agreement with previous findings (3). When correlations were examined with $L V$ rotational abnormalities, aortic elastic properties negatively correlated with LV apical rotation suggesting that increased aortic stiffness is associated with reduced LV apical rotation. These results are the opposite of those found in healthy subjects, where increased aortic stiffness showed positive correlations with LV apical rotation: the higher the aortic stiffness was, the higher the LV apical rotation was $(11,21)$. These results suggest that although normally directed LV rotational mechanics could be seen in most cases, not only reduced $\mathrm{LV}$ apical rotation could be detected, but the compensating effect of $L V$ rotational mechanics as seen in healthy subjects was absent in cTOF patients without LV-RBR. Although changes in $\mathrm{RV}$ dimensions are associated with abnormal $\mathrm{LV}$ flows and volumes suggesting significant interventricular interactions, the present study did not aim to examine in details (22).

The above mentioned results suggest several facts. In agreement with the recent findings, LV rotational mechanics can be non-invasively assessed by 3DSTE (7), for which normal reference values are also available (23). Although the results are contradictory with previous findings $(4,5)$, significant $L V$ rotational abnormalities could be detected in cTOF including LV-RBR and impairment of apical LV rotation. When different treatment strategies were compared, beneficial results could be found in patients with etrTOF corresponding with recent findings (24). Finally, abnormal physiologic response of LV rotational mechanics to increased aortic stiffness could be detected in cTOF patients without LV-RBR suggesting that the LV rotational mechanics have no ability to compensate for aortic stiffness. However, due to the facts that relatively low number of cTOF patients were examined and results are conflicting with current findings, further studies are warranted to confirm present results.

\section{Limitation section}

* A relatively small number of cTOF patients were evaluated in a single adult CHD center.

* LV rotational abnormalities were examined in adult cTOF patients. However, children with cTOF could 
show different results (25).

* 3DSTE has inferior temporal and spatial resolution when compared to $2 \mathrm{D}$ echocardiography, which could affect the results (7).

* The present study focused on the relationship between $\mathrm{LV}$ rotational abnormalities and aortic stiffness. Interventricular interaction was not evaluated by the current study (5).

* 3DSTE parameters are already validated, consequently, it was not aimed to validate them in this study. It was not purposed to perform detailed analysis of other heart chambers either.

* Echocardiographic measurement of aortic dimensions involves some inaccuracies, which could be taken into consideration when interpreting results.

\section{Conclusions}

Significant LV rotational abnormalities could be demonstrated in cTOF with high prevalence of LV-RBR. pcTOF patients showed significantly reduced LV apical rotation as compared to that of etrTOF cases. Unexpected abnormal physiologic response of $L V$ rotational mechanics to increased aortic stiffness can be detected in cTOF patients without $L V-R B R$.

\section{Acknowledgments}

Funding: None.

\section{Footnote}

Provenance and Peer Review: This article was commissioned by the Guest Editors (Yskert von Kodolitsch, Harald Kaemmerer, Koichiro Niwa) for the series "Current Management Aspects in Adult Congenital Heart Disease (ACHD): Part III" published in Cardiovascular Diagnosis and Therapy. The article has undergone external peer review.

Reporting Checklist: The authors have completed the STROBE reporting checklist. Available at http://dx.doi. org/10.21037/cdt-20-365

Data Sharing Statement: Available at http://dx.doi. org/10.21037/cdt-20-365

Conflicts of Interest: All authors have completed the ICMJE uniform disclosure form (available at http:// dx.doi.org/10.21037/cdt-20-365). The series "Current Management Aspects in Adult Congenital Heart Disease (ACHD): Part III" was commissioned by the editorial office without any funding or sponsorship. The authors have no other conflicts of interest to declare.

Ethical Statement: The authors are accountable for all aspects of the work in ensuring that questions related to the accuracy or integrity of any part of the work are appropriately investigated and resolved. Informed consent was given by both cTOF patients and control subjects. The institutional human research committee approved the study (project number: 71/2011), which complied with the Declaration of Helsinki (as revised in 2013).

Open Access Statement: This is an Open Access article distributed in accordance with the Creative Commons Attribution-NonCommercial-NoDerivs 4.0 International License (CC BY-NC-ND 4.0), which permits the noncommercial replication and distribution of the article with the strict proviso that no changes or edits are made and the original work is properly cited (including links to both the formal publication through the relevant DOI and the license). See: https://creativecommons.org/licenses/by-nc-nd/4.0/.

\section{References}

1. Apitz C, Webb GD, Redington AN. Tetralogy of Fallot. Lancet 2009;374:1462-71.

2. Ramaprabhu K, Idhrees M, Velayudhan B. Aortopathy in tetralogy of Fallot—a collective review. Indian J Thorac Cardiovasc Surg 2019;35:575-8.

3. Cruz C, Pinho T, Sousa C, et al. Ascending aorta in tetralogy of Fallot: Beyond echocardiographic dimensions. Echocardiography 2018;35:1362-9.

4. Menting ME, van den Bosch AE, Eindhoven JA, et al. Abnormal left ventricular rotation and twist in adult patients with corrected tetralogy of fallot. Eur Heart J Cardiovasc Imaging 2014;15:566-74.

5. Dragulescu A, Friedberg MK, Grosse-Wortmann L, et al. Effect of chronic right ventricular volume overload on ventricular interaction in patients after tetralogy of Fallot repair. J Am Soc Echocardiogr 2014;27:896-902.

6. Havasi K, Kalapos A, Berek K, et al. More than 50 years' experience in the treatment of patients with congenital heart disease at a Hungarian university hospital. Orv Hetil 2015;156:794-800.

7. Nemes A, Kalapos A, Domsik P, et al. Three-dimensional 
speckle-tracking echocardiography -- a further step in non-invasive three-dimensional cardiac imaging. Orv Hetil 2012;153:1570-7.

8. Lang RM, Badano LP, Mor-Avi V, et al. Recommendations for cardiac chamber quantification by echocardiography in adults: an update from the American Society of Echocardiography and the European Association of Cardiovascular Imaging. Eur Heart J Cardiovasc Imaging 2015;16:233-70.

9. Nemes A, Geleijnse ML, Forster T, et al. Echocardiographic evaluation and clinical implications of aortic stiffness and coronary flow reserve and their relation. Clin Cardiol 2008;31:304-9.

10. Nakatani S. Left ventricular rotation and twist: why should we learn? J Cardiovasc Ultrasound 2011;19:1-6.

11. Nemes A, Kalapos A, Domsik P, et al. Correlations between echocardiographic aortic elastic properties and left ventricular rotation and twist - Insights from the three-dimensional speckle-tracking echocardiographic MAGYAR-Healthy Study. Clin Physiol Funct Imaging, 2013;33:381-5.

12. van Dalen BM, Caliskan K, Soliman OI, et al. Left ventricular solid body rotation in non-compaction cardiomyopathy: A potential new objective and quantitative functional diagnostic criterion? Eur J Heart Fail 2008;10:1088-93.

13. Popescu BA, Beladan CC, Calin A, et al. Left ventricular remodelling and torsional dynamics in dilated cardiomyopathy: reversed apical rotation as a marker of disease severity. Eur J Heart Fail 2009;11:945-51.

14. Maharaj N, Khandheria BK, Peters F, et al. Time to twist: marker of systolic dysfunction in Africans with hypertension. Eur Heart J Cardiovasc Imaging 2013;14:358-65.

15. Nemes A, Földeák D, Domsik P, et al. Different patterns of left ventricular rotational mechanics in cardiac amyloidosis-results from the three-dimensional speckletracking echocardiographic MAGYAR-Path Study. Quant Imaging Med Surg 2015;5:853-7.

16. Kormányos Á, Domsik P, Kalapos A, et al. Left ventricular twist is impaired in acromegaly: Insights from the three-dimensional speckle tracking echocardiographic MAGYAR-Path Study. J Clin Ultrasound 2018;46:122-8.

17. Nemes A, Havasi K, Domsik P, et al. Left Ventricular
Rigid Body Rotation in Ebstein's Anomaly from the MAGYAR-Path Study. Arq Bras Cardiol 2016;106:544-5.

18. Nemes A, Havasi K, Domsik P, et al. Can univentricular heart be associated with "rigid body rotation"? A case from the three-dimensional speckle-tracking echocardiographic MAGYAR-Path Study. Hellenic J Cardiol 2015;56:186-8.

19. Nemes A, Havasi K, Forster T. "Rigid body rotation" of the left ventricle in hypoplastic right-heart syndrome: a case from the three-dimensional speckle-tracking echocardiographic MAGYAR-Path Study. Cardiol Young 2015;25:768-72.

20. van Grootel RWJ, van den Bosch AE, Baggen VJM, et al. The prognostic value of myocardial deformation in adult patients with corrected tetralogy of Fallot. J Am Soc Echocardiogr 2019;32:866-875.e2.

21. Zhang J, Chowienczyk PJ, Spector TD, et al. Relation of arterial stiffness to left ventricular structure and function in healthy women. Cardiovasc Ultrasound 2018;16:21.

22. Schafer M, Browne LP, Jaggers J, et al. Abnormal left ventricular flow organization following repair of tetralogy of Fallot. J Thorac Cardiovasc Surg 2020;160:1008-15.

23. Kormányos Á, Kalapos A, Domsik P, et al. Normal values of left ventricular rotational parameters in healthy adults - Insights from the three-dimensional speckle tracking echocardiographic MAGYAR-Healthy Study. Echocardiography 2019;36:714-21.

24. Nemes A, Kormányos Á, Havasi K, et al. Mitral annulus is enlarged and functionally impaired in adult patients with repaired tetralogy of Fallot as assessed by threedimensional speckle-tracking echocardiography-results from the CSONGRAD Registry and MAGYAR-Path Study. Cardiovasc Diagn Ther 2019;9:S221-7.

25. Karnik R, Uppu SC, Tozzi M, et al. Abnormalities in Left Ventricular Rotation Are Inherent in Young Children with Repaired Tetralogy of Fallot and Are Independent of Right Ventricular Dilation. Pediatr Cardiol 2018;39:1172-80.

Cite this article as: Nemes A, Rácz G, Kormányos Á, Domsik P, Kalapos A, Gyenes N, Ambrus N, Hartyánszky I, Bogáts $\mathrm{G}$, Havasi K. Left ventricular rotational abnormalities in adult patients with corrected tetralogy of Fallot following different surgical procedures (Results from the CSONGRAD Registry and MAGYAR-Path Study). Cardiovasc Diagn Ther 2021;11(2):623-630. doi: 10.21037/cdt-20-365 